\title{
DYNAMICS AND ORBITAL EVOLUTION OF OORT CLOUD COMETS
}

\author{
J.Q. ZHENG, M.J. VALTONEN AND S. MIKKOLA \\ Tuorla Observatory, University of Turku, Finland \\ AND \\ H. RICKMAN \\ Astronomical Observatory, Uppsala University, Sweden
}

Investigators generally conjecture a steady flux of new comets from the Oort cloud through the inner Solar system. Due to gravitational perturbations by major planets these objects may escape, become long period comets (LPCs) if their orbital periods $\mathrm{P}$ are larger than $200 \mathrm{yr}$ or become short period comets (SPCs) when their period is less than 200yr. SPCs are further divided in two types: the Halley type comets (HT, for $P>20 y r$ ) and the Jupiter family comets (JF, for $P<20 y r$ ).

The most striking characteristic of the observed SPCs is the great preponderance of low inclination prograde orbits especially for JF comets, in comparison with the roughly random distribution of orbital inclinations of the long period comets. To explain the origin of SPCs some investigators suggest a disk-like source, Kuiper belt or an inner Oort cloud.

The most direct way to attack the problem of orbital evolution of Oort cloud comets is to integrate the orbits of large numbers of comets up to the age of the Solar system. But the required computational effort is overwhelming unless the problem is somehow simplified.

In view of the difficulty with direct integrations, we develop a Monte Carlo method which is outlined by Valtonen et al. (1992). The method is based on cross-sections (energy change distribution) obtained with accurate orbit integrations using a KS-regularization. At the same time, we obtained the distribution functions for changes of orbital elements inclination $i$ and perihelion distance $q$ corresponding to each value of the energy change $\Delta \mathrm{E}$ and the values of the initial orbital elements $i_{0}$ and $q_{0}$ (see Zheng, 1994).

We assume that the orbit of a comet in the Solar system follows a constant ellipse for most of the time until occasionally the comet comes 
close to a planet and changes to a new orbit as a result of the gravitational influence of the planet. Then the comet keeps its new orbit approximately for several tens or hundreds of revolutions before the next close encounter.

In our Monte Carlo method, we first obtain the energy change of the comet using random sampling and the cross-sections which we have previously calculated, and then corresponding to the chosen value of the energy change we use the relative distribution functions of the new orbital elements and choose the new $i$ and the new $q$ of the cometary orbit also by random process.

Our starting point is the isotropic directional distribution of Oort cloud comets and the observed annual flux of new comets. We repeat the encounter process with four major planets and obtain new orbital elements for the comets until they leave the Solar system or become SPCs.

Generally in our simulation an Oort cloud comet makes several hundreds close encouters with major planets before it becomes a JF comet. But we find that the orbital evolution is dominated by a few very close encounters with large energy changes. We calculate the steady state population of the captured objects and record the orbital elements when they are such that the comets might be observed, i.e. if their $q<q_{l i m}$, where $q_{l i m}=1.5$ or 2.6 $\mathrm{AU}$ in our simulations. Another parameter in the simulations is the number of close approaches to the Sun $N_{\max }$ during the active lifetime of a comet. Here 'close approach' means $q<q_{\text {lim }}$. We choose $N_{\max }=400,1000$ or 4000 for different cases in our computations. After $N_{\max }$ close approaches to the Sun we assume that a comet becomes a 'dead' comet and it is left out of further calculation.

We have simulated several cases using different values of $q_{\min }$ and $N_{\max }$. In each case we calculate $N_{\text {model }}=1000000$ comets with initial perihelion distance $q_{0}$ between 0 and $30 \mathrm{AU}$ distributed uniformly or non-uniformly. In our simulations, the uniform $q_{0}$ distribution cannot produce enough JF comets. Therefore we suppose that the relative annual number $\mathrm{N}$ (per AU) of Oort cloud comets increases with $q_{0}$ as $N=1.0+0.014 \cdot q^{1.82}$ when $q<13 \mathrm{AU}$ and that it has a constant value of $N=5$ when $q>13 \mathrm{AU}$. This gives average value of $\mathrm{N}$ between $0-30 \mathrm{AU}$ of 3.48 .

For comparison with observations we have to know the real comet flux. The observed Oort cloud flux is 4.6 comets/AU/yr at the absolute magnitude $H_{10}=10.8$ and at $1 \mathrm{AU}$ (Bailey and Stagg 1988, Hughes 1988). But in our simulations, we only consider the encounters producing energy changes larger than a value of $\Delta E_{\min }=1 / 2000 A U^{-1}$ which means that only $1 / 6$ of all new comets are captured during the first step. In addition, since the flux is expected to be on the increase at present (Matese et al. 1995), we further divide the observed Oort cloud flux by 1.5 in order to get a representative average flux for the past few million years. Then we 
get the Oort cloud flux appropriate for comparison with the simulations of $N_{\text {new }}=0.5$ comets $/ \mathrm{yr} / \mathrm{AU}$ at $1 \mathrm{AU}$ and the total flux over the $q$-interved of $30 \mathrm{AU}$ of $N_{\text {flux }}=30 \mathrm{AU} \cdot 3.48 \cdot N_{\text {new }}$.

Finally, the population of JF comets is $N_{\text {flux }} \cdot T_{\text {life }} \cdot N_{J F} / N_{\text {model }}$, where active lifetime of JF comets $T_{\text {life }}$ is $P \cdot N_{\max }=8 \mathrm{yr} \cdot 400=3200 \mathrm{yr}$ (in case of $N_{\max }=400$ ), and $N_{J F} / N_{\text {model }}$ is the probability of an Oort cloud comet to become a JF comet in our simulations.

We obtain $N_{J F}=3044 \mathrm{JF}$ comets in our simulation with a non-uniform $q$-distribution and $N_{\max }=400$. Substituting this to the obove calculation we obtain the population of $508 \mathrm{JF}$ comets with $q<2.6 \mathrm{AU}$. The median value of $\cos i$ is 0.985 . Figure 1 shows the comparison between our simulation results and the observed inclination distribution of JF comets. Figure 2 shows the comparison for the $q$-distributions.

We summarize our results as follows:

(1) We agree with Fernández and Gallardo (1994) that single planet encounters by Jupiter cannot produce JF comets with a low inclination distribution from the Oort cloud comets. But multiple encounters could do that.

(2) The distribution function of energy changes is not symmetrical between negative energy changes and positive energy changes even for small changes. For that reason most comets with near $0^{\circ}$ inclinations drift inwards in the diffusion process while only very few comets drift inwards with near $180^{\circ}$ inclinations.

(3) Due to the small masses of the outer planets relative to Jupiter, the encounters with the outer major planets produce a strong selection of low inclination distribution of SPCs.

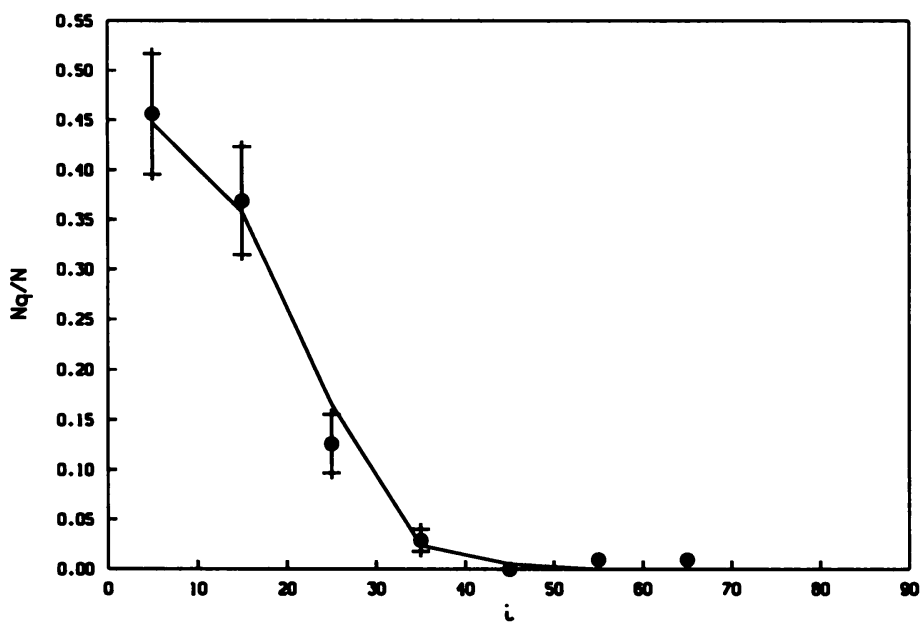

Fig. 1. The distribution of inclination $i$ of Jupiter family comets. Solid line: simulation results; dots with error bars: observations. 
(4) No HT comets with inclination less than $18^{\circ}$ or larger than $162^{\circ}$ have been observed (Marsden and Williams, 1994). It may be impossible for a retrograde comet to become a SP comet near Jupiter's orbital plane. But for low inclination orbit with $i<18^{\circ}$, the transfer process to a JF comet is one to two orders of magnitude faster than at the other inclinations. Therefore we may not see these comets when they are in the short-lived HT comets phase. Then we can say that the HT comets form a transfer source of JF comets from the Oort cloud.

In conclusion, we find it entirely possible that the SPCs are a captured subpopulation of the Oort cloud comets, and it is not necessary to assume the existence of additional comet sources to keep up the populations of the Jupiter family and the Halley type comets.

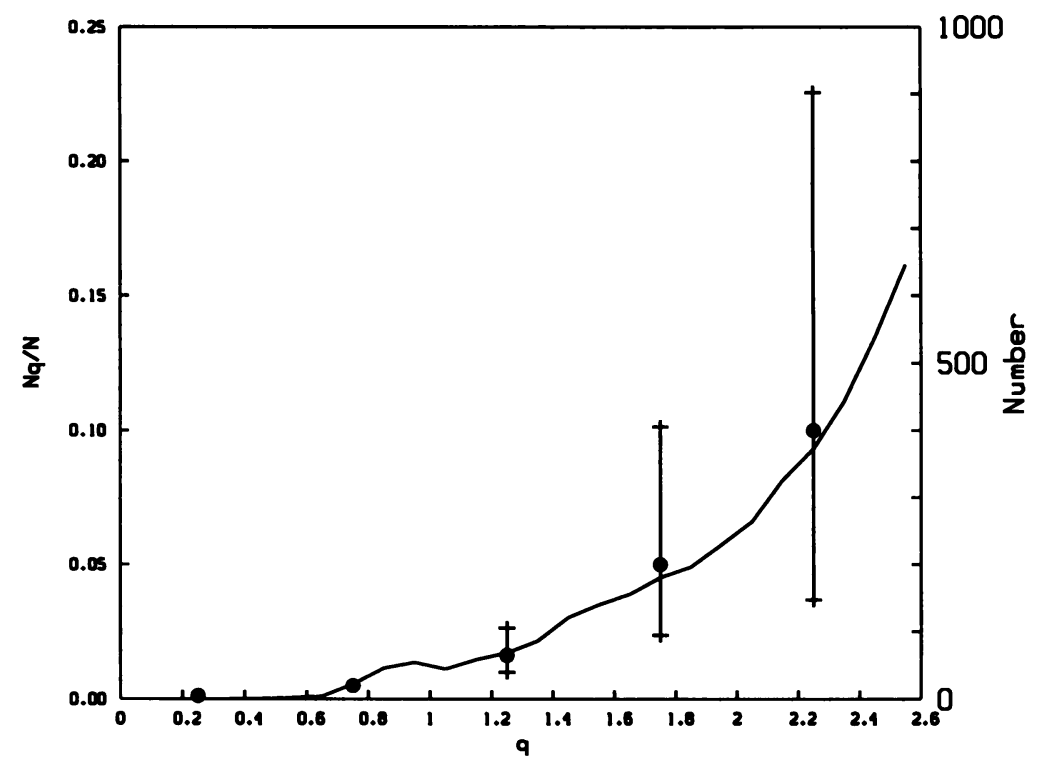

Fig. 2. The distribution of perihelion distance $q$ of Jupiter family comets. Solid line: simulation results; solid circles with error bars: extrapolated numbers from observations by Fernández et al.

\section{References}

Bailey, M.E. and Stagg, C.R.: (1988) MNRAS, 235, 1.

Fernández, J.A. and Gallardo, T.: (1994) $A \& A, 281,911$.

Hughes, D.W.: (1988) Icarus, 73, 149.

Marsden, B.G. and Williams, G.V.: (1994) Catalogue of Cometary Orbits, Smithsonian, pp. 80-83.

Matese, J.J., Whitman, P.G., Innanen, K.A. and Valtonen, M.J.: (1995) Icarus, 116, 255.

Valtonen, M.J., Zheng, J.-Q. and Mikkola, S.: (1992) Celestial Mech. 54, 37.

Zheng, J.-Q.: (1994) A $\mathscr{E}$ A Sup., 108, 253. 